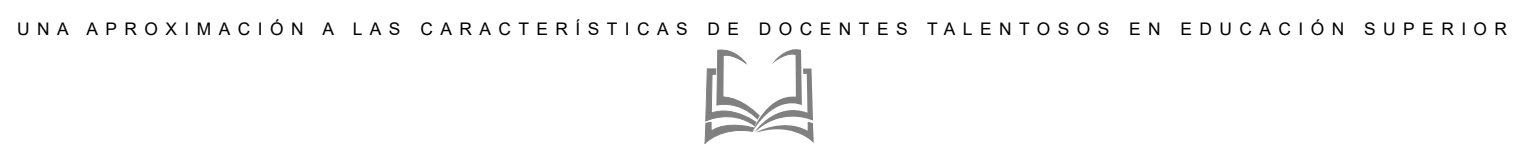

RECIBIDO EL 2 DE NOVIEMBRE DE 2019 - ACEPTADO EL 2 DE FEBRERO DE 2020

\title{
UNA APROXIMACIÓN A LAS CARACTERÍSTICAS DE DOCENTES TALENTOSOS EN EDUCACIÓN SUPERIOR
}

\section{AN APPROACH TO THE TALENTED CHARACTERISTICS OF TALENTED TEACHERS IN HIGHER EDUCATION}

\section{Diana Irasema Cervantes Arreola ${ }^{1}$}

\section{Alejandro Jesús Robles Ramírez ${ }^{2}$}

\section{Fabiola Lom Monárrez ${ }^{3}$}

Universidad Autónoma de Ciudad Juárez

\section{RESUMEN}

Esta investigación muestra un acercamiento a las características atribuidas de los estudiantes a los docentes talentosos en educación superior. El estudio es cuantitativo no experimental, transversal, de corte descriptivo, exploratorio y comparativo, con una muestra de 112 estudiantes de dos campus de una universidad pública del norte de México, de los cuales 70 $(62.5 \%)$ fueron mujeres y $42(37.5 \%)$ fueron hombres, con edades que oscilan entre los 18 y 35 años $(M=21, D E=3.12)$. Los resultados muestran que las características más atribuidas son: el dominio de contenidos, ser creativo, respetuoso, con comunicación asertiva, que promueve el desarrollo de sus estudiantes y brinda una explicación clara. Las relaciones

1 Doctora en Psicología, Maestra en Derechos Humanos, con Licenciatura en Psicología. Profesora de tiempo complete en el Departamento de Humanidades, Campus Sur, Universidad Autónoma de Ciudad Juárez, México. Tel. 6566882100-09, diana.cervantes@uacj.mx, ORCID 0000-0003-2353-1309. Miembro del SNI, investigadora del área de aptitudes sobresalientes y talento en jóvenes, talento docente y procesos de enseñanza-aprendizaje y docente de tiempo completo de la UACJ.

2 Maestro en Investigación educativ aplicada, Licenciado en Psicología. Profesor de catedra del Departamento de Humanidades, Campus sur, Universidad Autónoma de Ciudad Juárez, México. Tel.6566882100-09, jesus.robles@uacj.mx, ORCID 0000-0002-4560-9501. Investigador y catedrático del Centro de Actualización de Magisterio, Cd. Juárez y en la UACJ.

3 Maestra en Matemática educative, Licenciada en Matemáticas. Profesora de tiempo complete del Departamento de Ciencias Básicas, Campus Sur, Universidad Autónoma de Ciudad Juárez, México. Tel. 6566882100-09, fabiola.lom@uacj.mx, ORCID 0000-0002-2062-7068. Investigadora y docente de tiempo completo del área de ingeniería. 
que puntúan de manera más significativa con sexo y nivel que cursa el estudiante, son: ser responsable, dar acompañamiento en casos de rezago y el ser comprensivo.

PALABRAS CLAVE: docente talentoso, educación superior, características, elementos, relación.

\section{ABSTRACT}

This investigation shows the approach in the exploration in the characteristics attributed to the teacher's talent that the university's students. The study is quantitative non-experimental, transversal, descriptive, exploratory and comparative, with a sample of 112 students from two campus a public university in northern Mexico, of which 70 (62.5\%) were women and $42(37.5 \%)$ were men, with ages ranging between 18 and 35 years $(M=21, S D=3.12)$. The results show that the characteristics most attributed to the teacher's talent were: mastery of content, being creative, respectful, with assertive communication, which promotes the development of its students and provides a clear explanation. In significant relationships with student's gender and career level are: being responsible, provides accompaniment in cases of lag and being understanding.

KEYWORDS: teacher's talent, university, characteristics, elements, relation.

\section{INTRODUCCIÓN}

Las experiencias que denotan excepcionalidad en la enseñanza marcan el aprendizaje de los estudiantes, generando con ello una especie de evaluación sobre los docentes que son importantes en sus vidas y formación. Por estas razones un tema relevante en los procesos de enseñanza-aprendizaje y el ámbito educativo, son los docentes talentosos. Urbina (2014) menciona que, para estos procesos de enseñanza, se requiere pasión para poder llevar a cabo y de manera efectiva, los ejercicios pedagógicos; tal como lo es el sentimiento, la motivación y, por supuesto, el amor por el saber.

Hablar del talento docente no es sencillo, pues en la literatura sobre este tema no existe un concepto unánime, ya que se mencionan mayormente características, actitudes, aptitudes o elementos que un buen docente tiene, tal como lo describe Beltrán et al. (2015) pues "considera como parte importante de las características del buen docente la motivación, el liderazgo, la empatía, el espíritu emprendedor, la buena disposición, una actitud positiva, la estimulación intelectual, la comprensión, el sostén emocional y formador de subjetividades" (p. 41). Sin embargo, García-Cepero (2015) ha logrado generar una conceptualización de este término, donde explica que el talento docente se encuentra en personas que "han presentado un desempeño superior en la creación de oportunidades de aprendizaje excepcionales, al compararlos con sus pares" (parr.4). Dada esta definición, es importante destacar cómo es que estos docentes, con características específicas, ayudan a desarrollar estudiantes con grandes capacidades, con excelentes habilidades y con excepcionales herramientas para competir con sus iguales.

Estos docentes talentosos, que trabajan en la formación de individuos con grandes potencialidades, de acuerdo a López (2010) se retroalimentan por las experiencias de éxito que tienen con sus alumnos y por las proyecciones de su trabajo que no pasa inadvertido para los demás. El llevar un ritmo de crecimiento exponencial en los estudiantes y conducirlos al éxito, sin duda, tiene detrás a un docente talentoso. Así mismo, Guzmán (2013) considera como responsabilidad mutua al éxito, siempre teniendo en cuenta a los alumnos y sus padres en el desarrollo académico, permitiendo así, la optimización del éxito docente como complemento. 
Es entonces que las actividades docentes convergen directamente con su contexto, lo cual permite y direcciona el perfil de éxito de un docente "un dominio determinado por factores cognitivos, no cognitivos, personales, sociales y estocásticos cuyo sentido está en el desarrollo de condiciones por parte de la sociedad, para que el individuo pueda tener una vida prolongada, saludable y creativa, que a la vez contribuya al desarrollo de la comunidad" (García-Cepero, 2015, p. 9).

Por estas razones, el talento docente es una conjunción de variadas características, las cuales necesitan ser identificadas y que sin duda son generadoras de extraordinarios cambios en los estudiantes.

\section{EL TALENTO DOCENTE: UN MARCO REFERENCIAL}

Debido a los cambios recientes en la educación conforme a la necesidad de brindar innovaciones y entre estas un servicio educativo de calidad, el docente juega un papel importante en el desarrollo de la misma eficacia del sistema educacional (García-Cepero et al., 2016). Esto genera la necesidad de buscar, identificar y formar a docentes talentosos.

Pérez y Pérez (2015) formularon una investigación donde se buscaba implantar el modelo de selección finlandés en España, que consta de una prueba de aptitud, una entrevista evaluada por tres docentes y que buscaba la idoneidad para su formación como docente, la motivación y sus competencias comunicativas. Ello se aplicó a las personas aspirantes a entrar a formarse como educadores del nivel primario, en comparación con la tradicional Prueba de Acceso a la Universidad (PAU). Los resultados que se encontraron fueron que los estudiantes españoles presentaban algunos déficits importantes en la parte de las capacidades cognitivas de orden superior, como es el caso en, analizar, comparar, organizar el pensamiento, en argumentar y al proponer alternativas, así como un "déficit importante en relación a las disposiciones subjetivas que les llevan a decidir ser docentes" (Pérez y Pérez, 2015, p. 132) y finalmente también existían deficiencias en las capacidades de argumentación, transferencia y creación, en cuestiones de sensibilidad pedagógica ante posibles problemas educativos importantes y muy poca motivación en relación a los distintos requisitos, condiciones $y$ expectativas de lo que se genera o acontece en la función docente. Estas características son relevantes a la hora de enunciar lo que un profesor talentoso posee como competencias.

El talento docente puede ser identificado tempranamente, tal es el caso de las personas que reciben una evaluación, ya sea, que se aplique antes o después de que los estudiantes tomen cualquier curso de preparación para maestros. Esto, mostró que un rendimiento sólido en la evaluación para entrar a la facultad de educación predijo el desempeño exitoso de la enseñanza, confirmando que la aparición del talento se presenta antes de cualquier certificación educativa o el entrar propiamente a la universidad (Gordon, 2004). Aun así, es importante la retroalimentación por parte de los educandos, puesto que puede lograr reforzar el compromiso de los docentes en orden de mantener métodos de enseñanza efectivos (Choong, Hassan, Lee y Vadivelu, 2015).

García-Cepero et al. (2016), muestran que la experiencia es una característica de los docentes talentos, así como la capacidad de entender que los demás ya sean profesores pares, directivos e incluso los estudiantes pueden enseñarle o aprender a la par de ellos, ligado a este la humildad, que sean capaces de innovar en su propia práctica, "[...] la apertura (generación de espacios para ejercer la creatividad), acompañado de altos niveles de exigencia, pasión, trabajo en equipo, preocupación más allá de lo programático" ( $p$. 
103), finalmente, la existencia de un aprendizaje autorregulado donde la importancia de saberes pedagógico-didácticos, así como competencias investigativas, el uso de tecnología, tener el conocimiento de los estudiantes y el contexto en el que estos se desarrollan, resulta importante.

García-Cepero et al. (2016) plantean que el talento docente es una característica emergente y con procesos facilitadores, y las rasgos distintivos de dicho producto, son: que los maestros generan un compromiso social con su trabajo, son aquellos que se comprometen y cuestionan sobre su quehacer docente, proponiendo innovaciones, generan cambios en su persona en orden de no contradecirse y mostrar con el ejemplo en sus labores docentes, trabajan en equipo, comprenden que el trabajo es interdisciplinar, se disponen a aprender con sus estudiantes, a transformar la práctica en relación a la retroalimentación externa de expertos, colegas y muestran una disposición para capacitarse y a presentarse a concursos y eventos.

Figura 1. Características y componentes del talento docente.

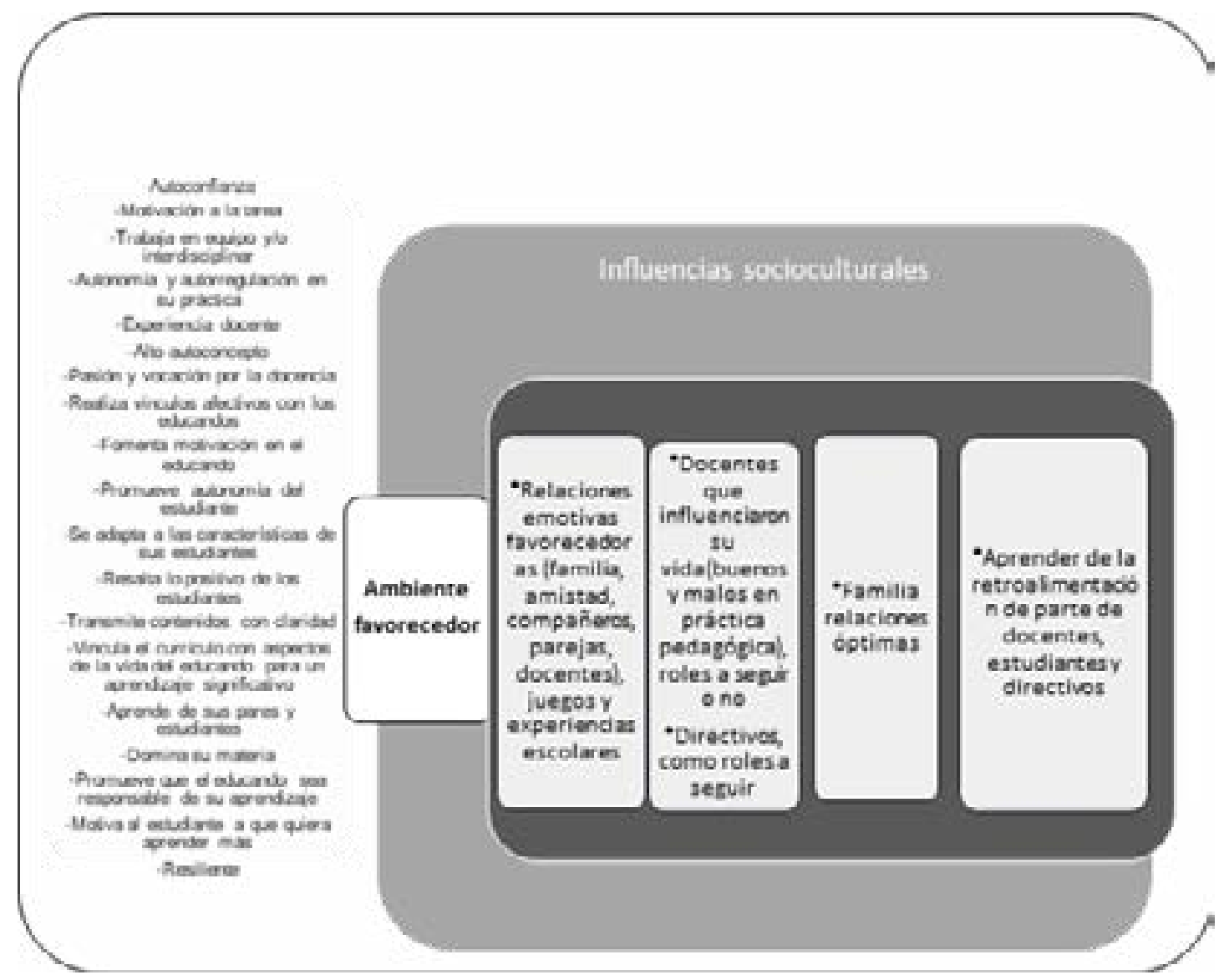

Fuente. Elaboración propia a partir de Branda y Porta (2012), Beltrán et al. (2015), Cortez et al. (2013), García-Cepero et al. (2016) Guzmán (2013), López (2010), Urbina (2014). 


\section{CONCEPCIÓN EXPLICATIVA DEL TALENTO BAJO UN MODELO SOCIOCULTURAL}

Para poder explicar la concepción del talento, se han tomado los modelos que conciben a la superdotación/aptitud sobresaliente, entendiendo que sobresaliente se define en varios modelos explicativos, en los cuales algunos de ellos no diferencian al talento de la aptitud sobresaliente o se usan de manera indistinta. García-Cepero et al. (2016) describe que "a finales del siglo XIX han surgido múltiples concepciones sobre a qué llamar talento y su relación con las capacidades excepcionales o la superdotación" (p. 89), sin embargo, en la actualidad no hay un consenso sobre la definición o término en relación a este tema, o este se puede concebir de acuerdo al modelo explicativo en que se enuncie, para poder establecer una diferencia entre términos, como lo han hecho autores contemporáneos.

En el caso de la concepción del talento docente, para este estudio se describen las características fundamentadas en relación al modelo sociocultural o histórico-social, bajo el Modelo estrella o psicosocial de Tannenbaum (1997), el cual refiere que los factores en los que la aptitud sobresaliente y el talento se denota son: (a) las habilidades generales (refieren al nivel intelectual), (b) las habilidades específicas(desde un punto de vista estático se pueden tener en diferentes áreas), (c) los factores no intelectuales (variables que influyen en la mejora de las habilidades: emocional o comportamental), (d) factores ambientales (contexto social, familia, escuela, cultura, comunidad) y (e) factores fortuitos(suerte, oportunidades o posibilidades que tiene la persona para lograr el éxito en sus metas) (Tannenbaum, 1986).

En este modelo Tannenbaum (1986) menciona que el talento es la combinación de diversos factores que darán como resultado algún talento en específico. Es entonces que el talento docente se entenderá como esa combinación de factores intelectivos, específicos, facilitadores (no intelectivos), ambientales y fortuitos que se manifiestan con un alto rendimiento y destacan para transformar y producir ideas ejemplares en los estudiantes y la sociedad.

Bajo esta última concepción, esta investigación refiere al talento docente desde la visión que de acuerdo a Blumen (2015) explicaba Tannenbaum, donde el verdadero talento se demuestra en la edad adulta, siendo personas con niveles de desempeño sobresaliente, como modelos que sean positivos para la sociedad o como creadores de ideas que transforman a la humanidad.

\section{MÉTODO}

\section{OBJETIVOS E HIPÓTESIS}

Este estudio no experimental y transversal de corte descriptivo, exploratorio y comparativo, pretende generar una aproximación para identificar cuáles son características de los docentes universitarios talentosos a partir de la perspectiva de los estudiantes y también conocer si existen diferencias significativas entre las características elegidas que perciben los estudiantes universitarios tiene un docente talentoso, de acuerdo al sexo, nivel de avance de la licenciatura y campus al que pertenecen. Se hipotetiza que existen diferencias significativas entre las características elegidas por parte de los estudiantes universitarios sobre el docente talentoso, de acuerdo al sexo, nivel de avance de la licenciatura y campus al que pertenecen.

\section{POBLACIÓN Y MUESTRA}

La muestra de este estudio fue no probabilística por conveniencia, de 112 estudiantes de nivel superior de campus sur y norte de una universidad pública del norte de México. De esta muestra, $23(20.5 \%)$ están matriculados en campus norte y $89(79.5 \%)$ en campus sur, pertenecientes 
a diferentes carreras: administración de empresas, psicología, contabilidad, educación, industrial y de sistemas, sistemas automotrices, física, sistemas computacionales, software y periodismo. De estos $70(63 \%)$ son mujeres y $42(37 \%)$ son hombres, con edades que oscilan entre los 18 y 35 años $(M=21, D E=3.12)$. La universidad establece tres niveles de avance dentro de la carrera, principiante, intermedio y avanzado, de los sujetos participantes, 33(30\%) son de nivel principiante, 37 (33\%) de intermedio y $42(37 \%)$ de avanzado.

\section{INSTRUMENTO}

La técnica de recolección de datos fue mediante una encuesta en línea denominada Características de docentes talentosos/ docentes de excelencia, de elaboración propia. Esta se constituyó en la primera parte del llenado de datos sociodemográficos: sexo y edad. Posteriormente datos de matriculación como: campus, programa/carrera y nivel actual de curso de la carrera. En el siguiente apartado contiene 48 enunciados, distribuidos en cinco apartados: a) conocimientos/atributos cognitivos, b) cualidades $y / o$ fortalezas personales, c) valores, d) comunicación y e) destrezas en la enseñanza, los cuales se respondieron seleccionando uno o más de los ítems, de acuerdo a cuáles de estas características consideraban los estudiantes que tiene un docente talentoso.

El apartado A de la encuesta se divide en nueve ítems: dominio de contenidos, conocimiento amplio de su materia o ramo, conocimiento de sus tareas y labores como docente, organización en sus ideas o trabajo, formación académica constante (cursos y/o posgrados), buscador/a de la verdad del conocimiento, explicación y trasmisión de sus ideas y su experiencia, reflexivo(a) y critico(a)/constructivo(a). El apartado B consta de 16 ítems: comprensivo(a), entusiasta, posee un concepto positivo de sí mismo(a), motivado(a), motivado(a) al logro creativo(a), autorregulado(a), autónomo, autoconfianza, orientador, sentido del humor, creador de vínculos de confianza, ordenado, tolerante a la crítica, paciente y generador de empatía. Del C con seis ítems: respetuoso, responsable, solidario, buscador de la justicia, ético y empático. La composición del rubro D con cuatro ítems: comunicación asertiva, comunicación clara y eficaz, diálogo motivacional y escucha abierta. El apartado D con 12 ítems: adecuada instrucción académica, promueve la autodirección y autonomía de desarrollo, despierta inquietud y cuestionamientos, promueve el desarrollo de sus estudiantes, facilitador intelectivo, búsqueda de nuevas posibilidades de aprendizaje, impulsa al trabajo colaborativo, utiliza múltiples estrategias pedagógicas, anima a ser responsable del propio desarrollo y aprendizaje, guía constantemente al estudiante para su aprendizaje, acompañamiento en casos de rezago y explicación clara de los contenidos de la clase.

Este instrumento fue validado por juicio de expertos y también se obtuvieron los valores de fiabilidad de alfa de Cronbach=.91 global y de cada uno de los apartados se obtuvo: Conocimientos/atributos cognitivos $(\alpha=.707)$, Cualidades $y / 0$ fortalezas personales $(\alpha=$ .758), Valores ( $\alpha=.626)$, Comunicación ( $\alpha=$ .337) y Destrezas en la enseñanza $(\alpha=.778)$. No hubo necesidad de eliminar el apartado de comunicación, debido a que no mejoraba ni afectaba el puntaje de los otros subapartados, ni global $<.01>$ y la escala total obtuvo un porcentaje bastante alto.

\section{PROCEDIMIENTO DE RECOGIDA Y ANÁLISIS DE DATOS}

La primera fase constó de localizar profesores de diversas carreras, tanto de campus sur y norte de la Universidad, que quisieran participar para invitar a sus grupos en responder la encuesta. Se realizó el envío de la encuesta por correo electrónico a los profesores que 
decidieron participar. Dicha encuesta contenía el consentimiento informado en la primera parte, para posteriormente responderse si así lo decidía el estudiante.

La segunda fase, fue la participación de los estudiantes en la encuesta, la cual se dio en un periodo de tres meses, donde las respuestas fueron almacenadas en una base de datos descargada en Excel, para su posterior análisis en el software SPSS Statistics 23.

Posteriormente para el análisis descriptivo de los datos, se utilizaron frecuencias y porcentajes de las características que se eligieron mayor número de veces en la encuesta. Después se hicieron pruebas ANOVA y Post-Hoc (Tukey), en orden de comparar las medias de las características más nombradas dentro del instrumento y encontrar si existían diferencias significativas.

\section{CONSIDERACIONES ÉTICAS}

Los participantes fueron informados del propósito del estudio, beneficios y repercusiones que pudiera tener la colaboración en la investigación, la confidencialidad de los datos que se proporcionaron, así como la duración de esta; dicha participación fue aceptada de manera voluntaria por los participantes.

\section{RESULTADOS}

En los resultados obtenidos para responder al primer objetivo sobre las características que perciben los estudiantes universitarios en un docente talentoso, en el análisis de frecuencias sobre los conocimientos y/o atributos cognitivos, señalan como la característica más enunciada, el dominio de contenidos (96\%), posteriormente conocimiento amplio de su materia o ramo (82\%) y crítico/ constructivo (74\%), este último casi a la par de organización en sus ideas y trabajo (72\%) y explicación y trasmisión de sus ideas y su experiencia $(71 \%)$. La figura 2 muestra las respuestas obtenidas sobre las características del apartado A de la encuesta.

Figura 2. Número de respuestas obtenidas sobre las características de conocimiento y atributos cognitivos del docente talentoso.

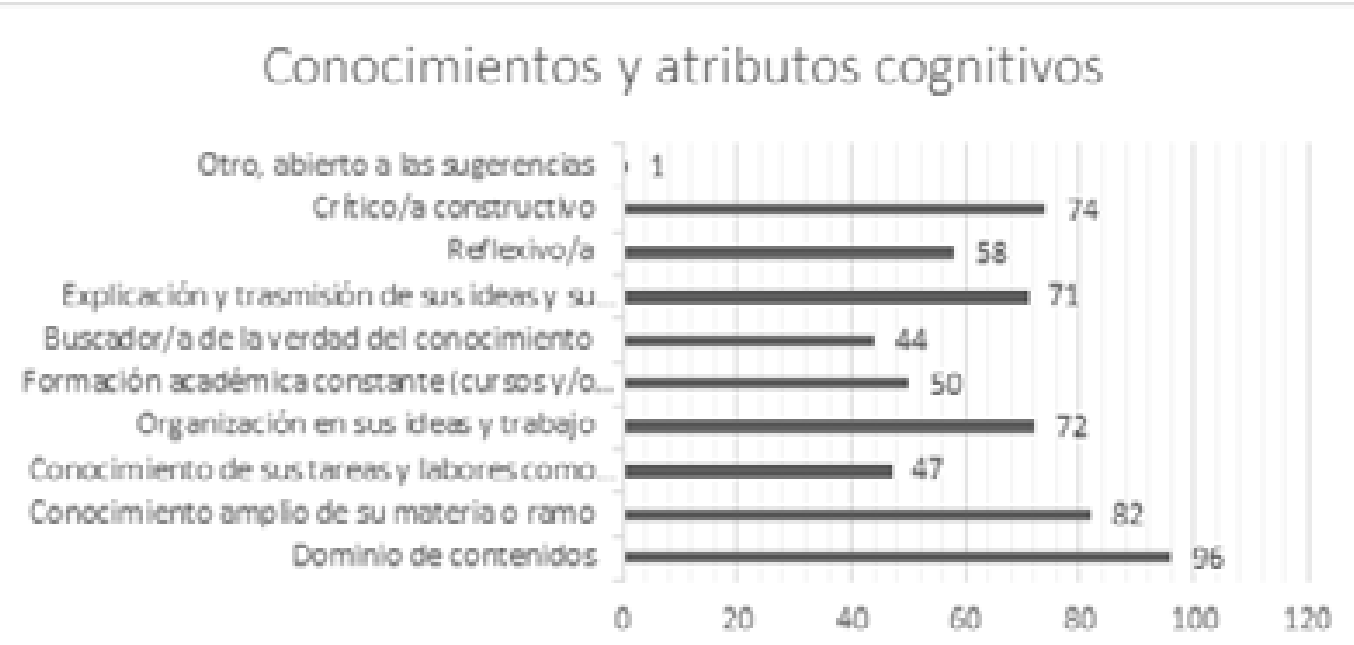

Fuente. Elaboración propia. 
En lo referente a Cualidades y/o fortalezas personales, los resultados muestran que los profesores talentosos son creativos $(78 \%)$, y en segunda instancia se encuentran en un mismo porcentaje las características de que son motivados $(72 \%)$ y comprensivos $(72 \%)$, otro punto señalado en un porcentaje muy similar es el ser paciente (71\%), quedando de manera subsecuente las características de que es tolerante a la crítica (63\%), genera empatía con los estudiantes (59\%), entusiasta (54\%) y posteriormente las demás cualidades con porcentajes más bajos al $54 \%$ (Figura 3 ).

Figura 3. Número de respuestas obtenidas sobre las características de cualidades y/o fortalezas del docente talentoso.

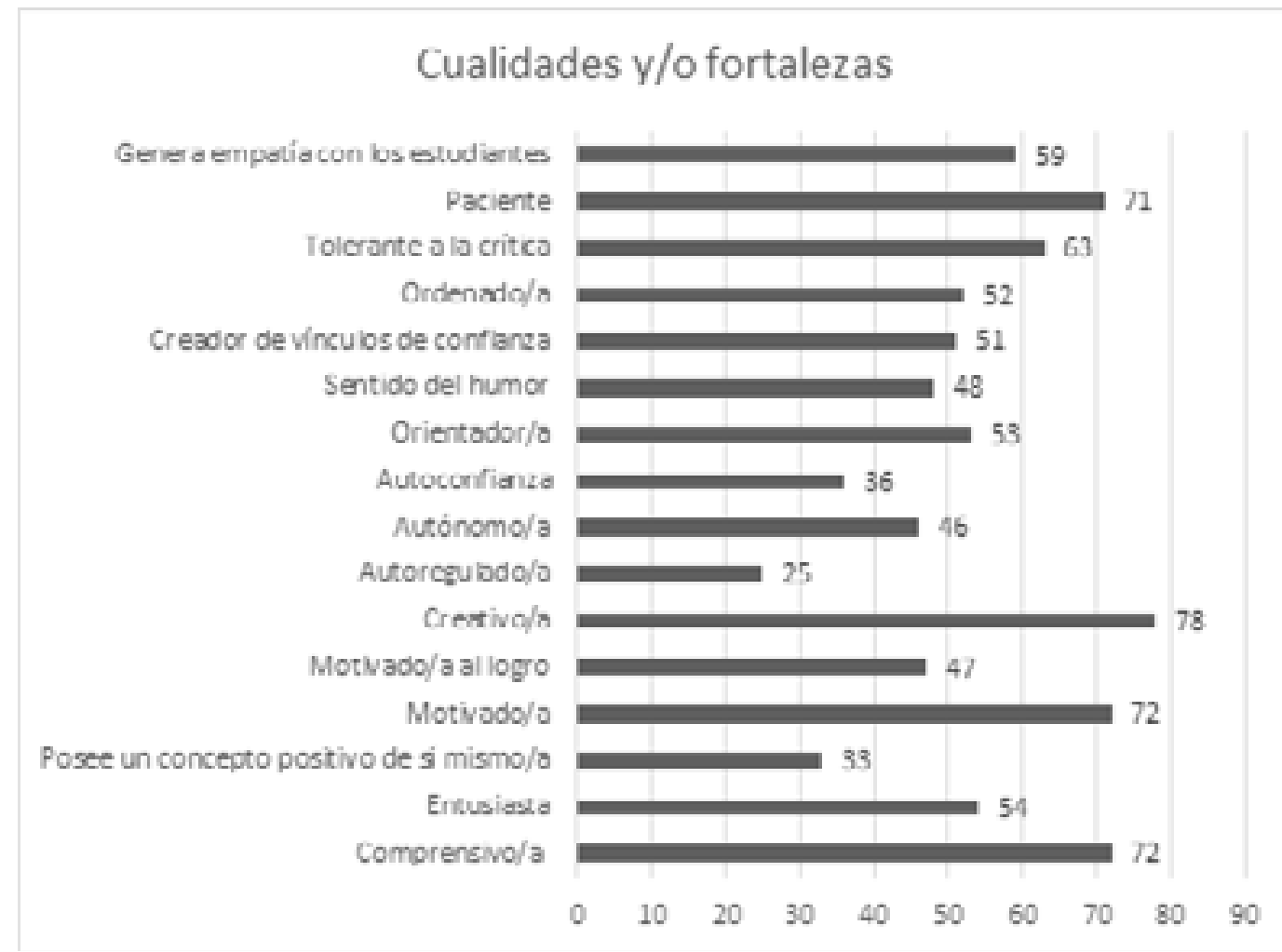

Fuente. Elaboración propia.

De los valores que los estudiantes consideran son una característica importante en el talento docente, es el ser respetuoso (93\%) y responsable $(90 \%)$, la ética $(79 \%)$ es otro valor relevante y la empatía (67\%) (Figura 4). 
Figura 4. Número de respuestas obtenidas sobre las características de valores del docente talentoso

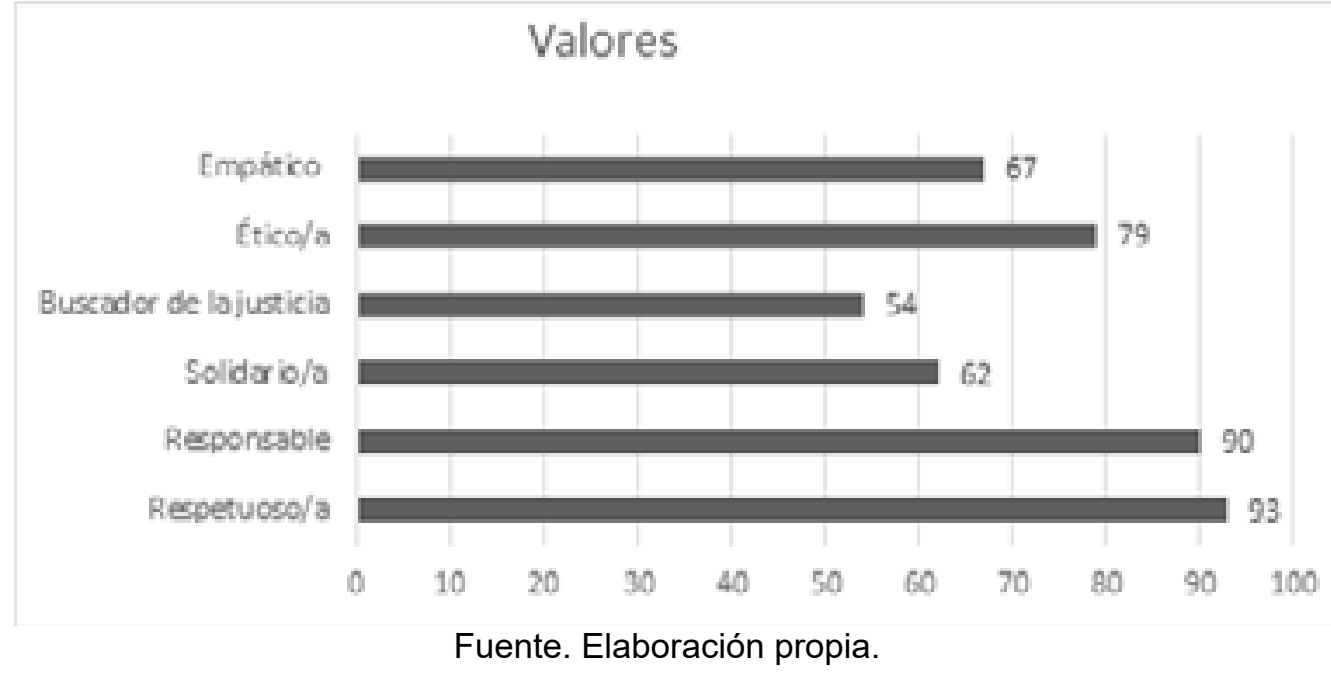

En las características sobre los aspectos de comunicación, los estudiantes señalaron como principal el tener una comunicación asertiva (88\%), una comunicación clara y eficaz (82\%), posteriormente la escucha abierta $(63 \%)$ y el tener un diálogo motivacional (48\%) (Figura $5)$.

Figura 5. Número de respuestas obtenidas sobre las características de comunicación del docente talentoso.

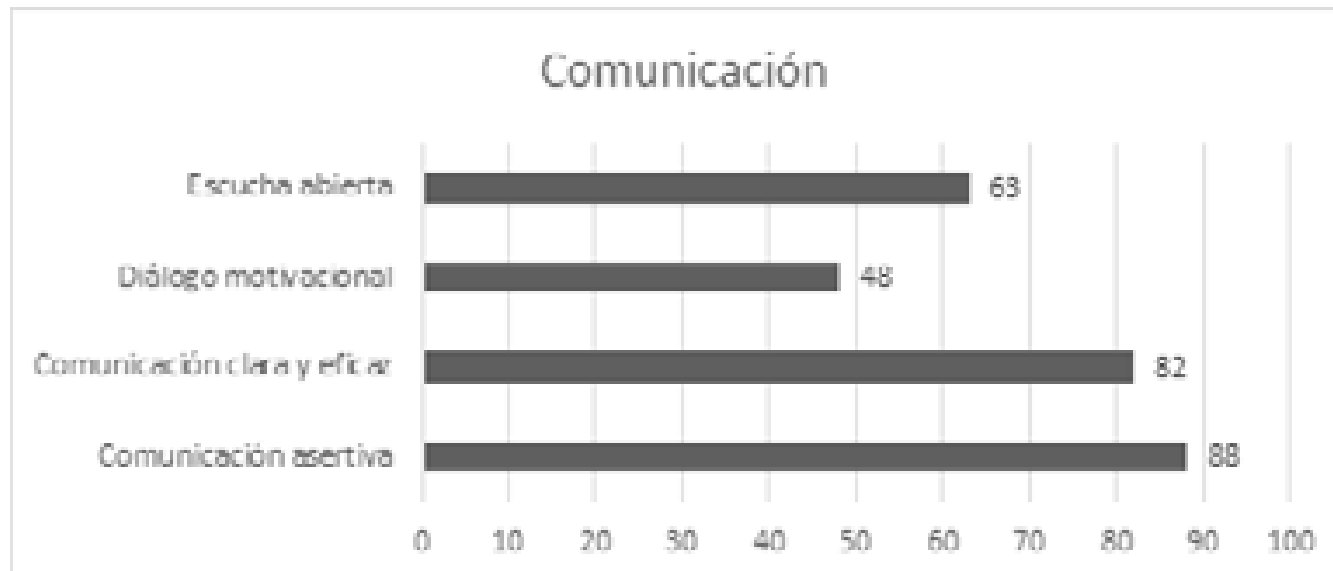

Fuente. Elaboración propia.

Las características que se denotan con mayor frecuencia en relación a las destrezas en la enseñanza son: promueve el desarrollo de sus estudiantes $(77 \%)$, explicación clara de los contenidos de clase $(75 \%)$, a la par en frecuencia una adecuada instrucción académica $(70 \%)$ y el que despierta inquietud y cuestionamientos (interés investigativo) (70\%). En otras características con una misma mención, el que guía constante del estudiante para su aprendizaje (63\%) y la búsqueda de nuevas posibilidades de aprendizaje (63\%). 
Con porcentajes de frecuencia que oscilan entre el $60 \%$ y el $46 \%$ se encuentran las demás características (Figura 6).

Figura 6. Número de respuestas obtenidas sobre las características de destrezas en la enseñanza del docente talentoso.

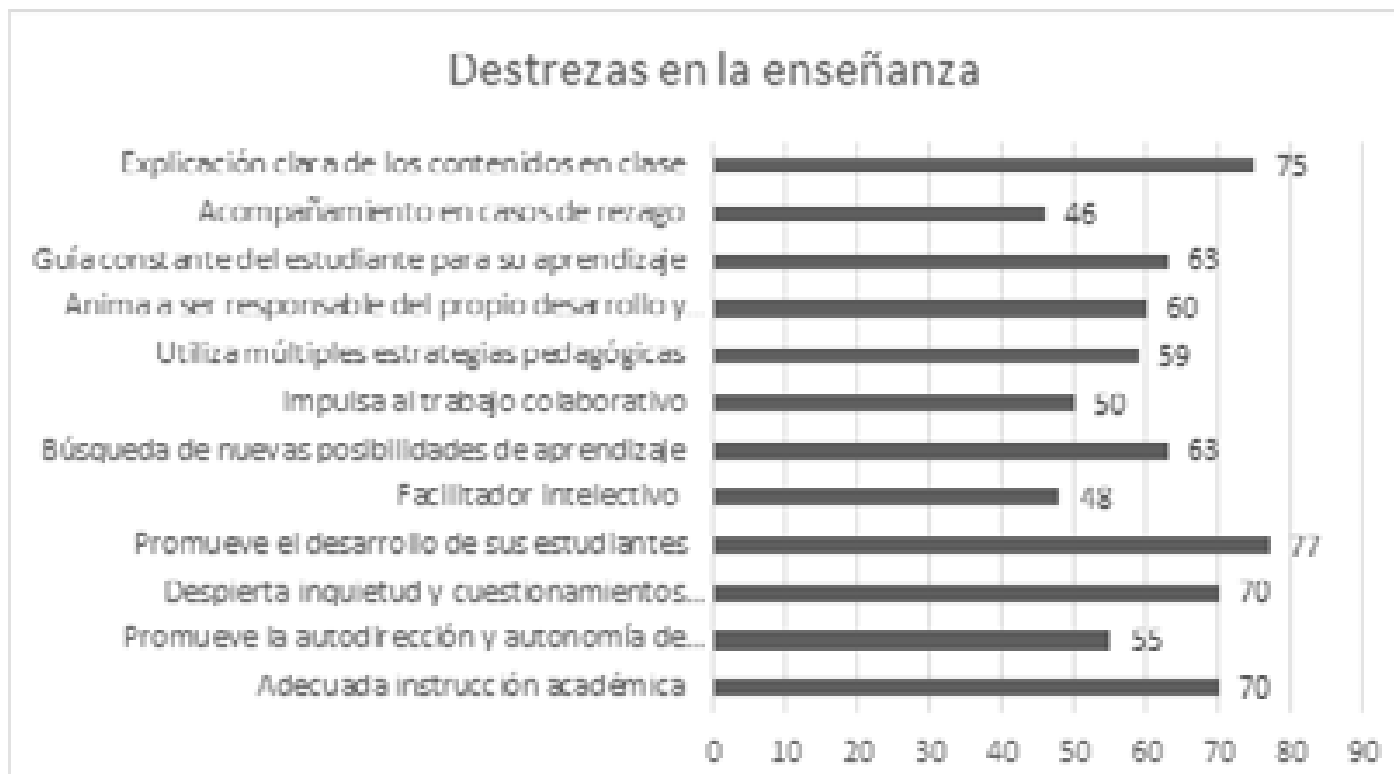

Fuente. Elaboración propia.

Con estos mismos datos, se procedió con la realización de pruebas de ANOVA, dividiendo en grupos mediante el sexo, nivel de avance de la licenciatura y campus nortesur, donde no se encontraron diferencias estadísticamente significativas, al comparar las medias entre campus sur y norte. Por otro lado, conforme al sexo, se encontró que, en la característica de entusiasta $(p<.012)$, las mujeres $(M=1.47, D E=.503)$ puntuaron más bajo que los hombres ( $\mathrm{M}=1.71, \mathrm{DE}=.457)$ en que los docentes talentosos tienen esta característica, con un ANOVA $(g l=111)=27.563$, como se puede ver en la tabla 1 obteniendo una $\mathrm{d}$ de Cohen= 0.49 es un tamaño de efecto acercándose a mediano, tomando en cuenta que, $d=<.02$ pequeño; $d=>.50$ mediano y $d=>.80$ grande (Cohen, 1988).

Tabla 1. Resumen de ANOVA entre sexos, conforme a la característica entusiasta

\begin{tabular}{ccccccccc} 
Característica & Sexo & $\mathbf{n}$ & $\mathbf{M}$ & $\mathbf{D E}$ & $\begin{array}{c}\text { Límite } \\
\text { inferior }\end{array}$ & $\begin{array}{c}\text { Límite } \\
\text { superior }\end{array}$ & gl & Sig. \\
\hline Entusiasta & Mujer & 70 & 1.47 & .503 & 1.35 & 1.59 & & \\
& Hombre & 42 & 1.71 & .457 & 1.57 & 1.86 & 111 & .012 \\
Total & & 112 & 3.18 & .96 & 2.92 & 3.45 & 111 & \\
\hline
\end{tabular}

Fuente. Elaboración propia 
Otra, característica que tuvo diferencias estadísticamente significativas, fue la de responsable $(\mathrm{p}<.011)$ con un ANOVA $(g l=111)$ $=9.920$, donde las mujeres $(\mathrm{M}=1.96, \mathrm{DE}=.204)$ mencionaron estar más de acuerdo los hombres
$(\mathrm{M}=1.81, \mathrm{DE}=.397)$, en que un docente con talento posee esta aptitud, esto se puede apreciar dentro de la tabla 2, se obtuvo una d de Cohen $=0.47$, nuevamente el efecto esta aproximado al mediano.

Tabla 2. Resumen de ANOVA entre sexos, conforme a la característica responsable

\begin{tabular}{ccccccccc} 
Característica & Sexo & $\mathbf{n}$ & $\mathbf{M}$ & DE & $\begin{array}{c}\text { Límite } \\
\text { inferior }\end{array}$ & $\begin{array}{c}\text { Límite } \\
\text { superior }\end{array}$ & gl & Sig. \\
\hline Responsable & Mujer & 70 & 1.96 & .204 & 1.91 & 2.01 & & \\
& Hombre & 42 & 1.81 & .397 & 1.69 & 1.93 & 111 & .011 \\
\hline \multirow{2}{*}{ Total } & & 112 & 3.77 & .601 & 3.6 & 3.94 & & \\
\hline
\end{tabular}

Fuente. Elaboración propia

Otra capacidad donde se encontraron diferencias estadísticamente significativas fue el utilizar múltiples estrategias pedagógicas $(\mathrm{p}<.022)$ [tabla 3] con un ANOVA ( $g /=111)$ $=27.107$, en la cual, los hombres $(M=1.45$,
$\mathrm{DE}=.504)$ mencionaron menos veces que las mujeres ( $M=1.67, D E=.473)$, en los docentes extraordinarios manejan múltiples estrategias durante su catedra, nuevamente el tamaño de efecto es casi mediano ( $d$ de Cohen=. 45).

Tabla 3. Resumen de ANOVA entre sexos, conforme a si el docente talentoso utiliza múltiples estrategias pedagógicas

\begin{tabular}{lcccccccc} 
Característica & Sexo & $\mathbf{n}$ & $\mathbf{M}$ & $\mathbf{D E}$ & $\begin{array}{c}\text { Límite } \\
\text { inferior }\end{array}$ & Límite superior & gl & Sig. \\
\hline Utiliza & Mujer & 70 & 1.67 & .473 & 1.56 & 1.78 & 111 & .022 \\
múltiples & Hombre & 42 & 1.45 & .504 & 1.30 & 1.61 & & \\
estrategias & & & & & & & & \\
$\begin{array}{l}\text { pedagógicas } \\
\text { Total }\end{array}$ & & & & & & & & \\
\hline
\end{tabular}

Fuente. Elaboración propia

Desde otra perspectiva, en la tabla 4 se puede observar que al comparar las medias dentro de los tres niveles de avance de la carrera (principiante, intermedio y avanzado), se encontró que comprensivo $(p<.023)$ con un ANOVA $(g l=111)=21.964$, los que están en el nivel principiante $(\mathrm{M}=1.58, \mathrm{DE}=.502)$ puntuaron más bajo que los de intermedio $(M=1.73$, $\mathrm{DE}=.450$ ) y propiamente tanto los de principiante e intermedio puntuaron más bajo que los que están en avanzado ( $\mathrm{M}=1.86, \mathrm{DE}=.354)$, tamaños de efecto se observan en la tabla 5 , en la cual se percibe que existe un tamaño de efecto bajo-mediano entre los principiantes y los intermedios, y un efecto medio-grande entre los de principiante y avanzado. Por otra parte, entre los intermedios y los de avanzados, se aprecia un efecto medio-grande. 
Tabla 4. Resumen de ANOVA entre niveles de avance de estudios, conforme a la característica comprensivo

\begin{tabular}{ccccccccc} 
Característica & Nivel & $\mathbf{n}$ & $\mathbf{M}$ & $\mathbf{D E}$ & $\begin{array}{c}\text { Límite } \\
\text { inferior }\end{array}$ & $\begin{array}{c}\text { Límite } \\
\text { superior }\end{array}$ & gl & Sig. \\
\hline Comprensivo(a) & Principiante & 33 & 1.58 &, 502 & 1.40 & 1.75 & & \\
& & & & & & & 111 & .023 \\
& Intermedio & 37 & 1.73 & .450 & 1.58 & 1.88 & & \\
& Avanzado & 42 & 1.86 & .354 & 1.75 & 1.97 & & \\
\multicolumn{2}{c}{ Total } & 112 & 4.87 & 1.306 & 4.73 & 5.6 & & \\
\hline
\end{tabular}

Fuente. Elaboración propia

Tabla 5. $d$ de Cohen conforme a la capacidad de ser comprensivo de los docentes

\begin{tabular}{llll} 
& Principiante X1 & Intermedio X2 & Avanzado X3 \\
\hline Principiante X1 & N/A & .31 & .64 \\
Intermedio X2 & .31 & N/A & .32 \\
\hline
\end{tabular}

Fuente. Elaboración propia

Otra característica destacada fue la de acompañamiento en casos de rezago $(\mathrm{p}<.027)$, con un ANOVA $(g /=111)=27.857$, donde, los principiantes $(\mathrm{M}=1.42, \mathrm{DE}=.502)$ puntuaron más altos que los de nivel intermedio ( $M=1.32$, $\mathrm{DE}=.475$ ) en que los decanos talentosos realizan esta actividad, pero, puntuaron más bajos que los de avanzado $(M=1.62, D E=.492)$, esto se puede corroborar en la tabla 6 y las $d$ de Cohen se aprecian en la tabla 7, donde el tamaño de efecto entre principiantes e intermedios fue bajo, pero entre principiantes y avanzados fue bajo-mediano, finalmente, entre intermedios y avanzados, fue mediano-alto. La prueba Post-Hoc de Tukey, se puede apreciar en la tabla 8 tanto de los ANOVA de comprensivo y Acompañamiento en casos de rezago.

Tabla 6. Resumen de ANOVA entre niveles de avance de estudios, conforme a la característica Acompañamiento en casos de rezago

\begin{tabular}{ccccccccc} 
Característica & Sexo & $\mathbf{n}$ & $\mathbf{M}$ & $\mathbf{D E}$ & $\begin{array}{c}\text { Límite } \\
\text { inferior }\end{array}$ & $\begin{array}{c}\text { Límite } \\
\text { superior }\end{array}$ & gl & Sig. \\
\hline $\begin{array}{c}\text { Acompañamiento } \\
\text { en casos de } \\
\text { rezago }\end{array}$ & Principiante & 33 & 1.42 &, 502 & 1.25 & 1.60 & & \\
& & & & & & & 111 & .027 \\
& Intermedio & 37 & 1.32 & .475 & 1.17 & 1.48 & & \\
Total & Avanzado & 42 & 1.62 & .492 & 1.47 & 1.77 & & \\
\hline
\end{tabular}

Fuente. Elaboración propia. 
Tabla 7. $d$ de Cohen conforme a la capacidad de atender casos de educandos en rezago

\begin{tabular}{llll} 
& Principiante X1 & Intermedio X2 & Avanzado X3 \\
\hline Principiante X1 & N/A & .20 & .40 \\
Intermedio X2 & .20 & N/A & .62 \\
\hline
\end{tabular}

Fuente. Elaboración propia

Tabla 8. Prueba Post-Hoc (Tukey) de ANOVA entre niveles de avance de estudios, referente a si los docentes talentosos poseen las características Comprensivo(a) y acompañamiento en casos de rezago

\begin{tabular}{|c|c|c|c|c|c|c|c|}
\hline Característica & $\begin{array}{l}\text { (I) Nivel de } \\
\text { estudios } \\
\text { en que se } \\
\text { encuentra }\end{array}$ & $\begin{array}{l}\text { (J) Nivel de } \\
\text { estudios } \\
\text { en que se } \\
\text { encuentra }\end{array}$ & $\begin{array}{l}\text { Diferencia } \\
\text { entre } \\
\text { medias }\end{array}$ & $\begin{array}{l}\text { Desv. } \\
\text { Error }\end{array}$ & Sig. & $\begin{array}{l}\text { LLímite } \\
\text { inferior }\end{array}$ & $\begin{array}{l}\text { Límite } \\
\text { superior }\end{array}$ \\
\hline \multirow[t]{6}{*}{ Comprensivo(a) } & Principiante & Intermedio & -.154 & .104 & .303 & -.40 & .09 \\
\hline & & Avanzado & -.281 & .104 & .017 & -.52 & -.04 \\
\hline & Intermedio & Principiante & 154 & .104 & .303 & -.09 & .40 \\
\hline & & Avanzado & -.127 & .098 & .397 & -.036 & .10 \\
\hline & Avanzado & Principiante & .281 & .101 & .397 & .04 & .52 \\
\hline & & Intermedio & .127 & .098 & .017 & -.10 & .38 \\
\hline \multirow{6}{*}{$\begin{array}{c}\text { Acompañamiento } \\
\text { en casos de } \\
\text { rezago }\end{array}$} & Principiante & Intermedio & .100 & .117 & .671 & -.18 & .38 \\
\hline & & Avanzado & -.195 & .114 & .205 & -.47 & .08 \\
\hline & Intermedio & Principiante & -.100 & .117 & .671 & -.38 & .18 \\
\hline & & Avanzado & -.295 & .110 & .023 & -.56 & -.03 \\
\hline & Avanzado & Principiante & .195 & .114 & .205 & -.08 & .47 \\
\hline & & Intermedio & .295 & .110 & .023 & .03 & .56 \\
\hline
\end{tabular}

Fuente. Elaboración propia

\section{DISCUSIÓN Y CONCLUSIONES}

Existe una preocupación constante sobre investigar y trabajar los temas relacionados a la docencia universitaria (Morán, 2004), entre ellos el conocer más sobre las características que definen el perfil de un profesor talentoso. Las interrogantes son muchas, sobre los procesos o forma de trabajo, estilo de enseñanza o experiencias y conocimientos que pueden ser factores que marquen una diferencia en la docencia talentosa, por ejemplo, el preguntarse sobre quién puede ser un docente con talento, si quienes estudian como profesión el ser profesor y/o los profesionales que estudian profesiones ajenas a la educación, pero que tal vez presentan habilidades y competencias que aunque no se tenga la formación como docente destacan.

Es entonces importante pensar sobre las características que pueden crear las diferencias entre si la formación o las competencias son componentes del talento docente, aunado a ello, Gordon (2004) quien refiere que el talento 
es intangible y está separado de las habilidades y los conocimientos, menciona que es el "arte de enseñar" (p.2) y que este puede ser identificado de manera temprana para los casos de quienes quieren o comienzan a dedicarse a la docencia. Por estas razones las características obtenidas en este estudio denotan que un conjunto de elementos conforma el talento docente, como lo es el ser responsable, dominar contenidos, ser creativo y motivado, respetuoso, responsable, entusiasta, con comunicación clara y eficaz, además asertiva, promover el desarrollo de sus estudiantes, el usar múltiples estrategias pedagógicas, a la par de ser comprensivo y brindar una explicación clara (pedagógico).

Este acercamiento exploratorio y descriptivo, fortalece en gran parte a los datos generados en algunas investigaciones (Branda y Porta, 2012, Beltrán et al., 2015, Cortez et al., 2013, GarcíaCepero et al., 2016, Guzmán, 2013, López, 2010 y Urbina, 2014) que hablan de características de experiencias de éxito docente, talento docente o lo que refleja a un buen docente y sobre los aspectos fundamentales que deben de aparecer en personas que quieren acceder a ser docentes. Ejemplo de estos aspectos, tiene que ver la buena comunicación, pues según Pérez y Pérez (2015) son la motivación hacia el campo de la educación y las habilidades y competencias en su comunicación, un elemento del buen docente, dato muy similar a lo obtenido en el caso de esta investigación. A la par de esto, para algunos de los estudiantes el acompañamiento en casos de rezago, el ser comprensivo, la responsabilidad y el usar diferentes estrategias, resultan características del docente talentoso.

Las relaciones significativas, encontradas en con el ser responsable, las mujeres están más de acuerdo con esa característica, en relación a lo que los hombres respondieron, cosa distinta a la característica de entusiasta, donde las mujeres puntuaron más bajo y los hombres más alto al denominarla como parte del talento docente. Con estos resultados también se puede observar que de las características de que el docente talentoso da acompañamiento en casos de rezago y el ser comprensivo, de acuerdo al nivel que están cursando en la carrera, las relaciones que puntúan de manera más significativa, se encuentran las que dan los estudiantes de avanzado. Esto da cuenta de que el tener destrezas en la enseñanza, dar acompañamiento a quienes lo requieren y comprenderlos, ayuda a que se promuevan y potencien experiencias de aprendizaje exitosas; pues estos docentes creen que el estudiante puede aprender, así como lo menciona Guzmán (2013).

Este estudio tiene algunas limitaciones, una de ellas se encuentra en el instrumento, el cual puedo generar mayor análisis de las respuestas, si se hubiera diseñado con una escala tipo Likert, otra se encuentra en el alcance de una mayor muestra, esto si se hubiera aplicado de manera personal y no en línea. Sin embargo, el aporte de este estudio abre más un campo, poco explorado, para dar la debida importancia a esos casos de docentes que muestran conductas de éxito dentro de sus aulas de clase, de sus escuelas y de sus acciones hacia los estudiantes, pues se sabe actualmente más sobre cómo trabajan o aprenden los estudiantes, pero no se ha explorado el otro actor en este proceso, como los docentes que son talentosos en su labor, por ello se sugiere realizan más estudios y a profundidad sobre características o elementos en estos casos.

\section{REFERENCIAS BIBLIOGRÁFICAS}

Beltrán, C., Cortés, S., Iriarte, I., Lozano, J., Sánchez, L., Martínez, J., Montero, V., Rojas, G., Rojas, J, Rodríguez, Z. y Vélez, A. (2015). Hacia una comprensión del talento docente (Tesis de maestría). Pontificia Universidad Javeriana. Bogotá DC, Colombia. Recuperada de file:///C:/Users/mante/ 
Downloads/MedinaNovaAdrianaAlejandra2016. pdf

Blumen, S. (2015). Abraham Tannenbaum: 1924-2014. Revista de Psicología (PUCP), 33(1), 231-234.

Cohen, J. (1988). Statistical Power Analysis for the Behavioral Sciences. Nueva York: Psychology Press.

Choong, C., Hassan, H., Lee, S., y Vadivelu, J. (2015). Using students' formative feedback to advocate reflective teaching. Medical education, 49(5), 535535. doi 10.1111/medu.12697

García-Cepero, M. (julio 10, 2015). Tiene sentido hablar de talento docente. Compartir Palabra Maestra. Recuperado de https:// compartirpalabramaestra.org/columnas/ tiene-sentido-hablar-de-talento-docente

García-Cepero, M., Gómez-Hernández, F., Barrios-Martínez, D., Santamaría, A., Castro Fajardo, L., Sánchez, A., y Zuluaga, Z. (2016). Itinerarios, hitos y catalizadores asociados a la emergencia del talento docente. Revista de Psicología(PUCP), 34(1), 85-115.

Gordon, G. (2004). Talent vs. training: Preparing tomorrow's teachers. The Gallup Poll Tuesday Briefing. Recuperado de https://www.gallup.com/topic/category_ education.aspx

Guzmán, C. (2013). Profesores ejemplares de establecimientos educativos públicos de educación secundaria y creencias pedagógicas: desafíos y propuestas para lamejoradeladocencia. Revistamexicana de investigación educativa, 18(58), 871-892. Recuperado de http://www. scielo.org.mx/scielo.php?script $=$ sci_ arttext\&pid=S1405-66662013000300009
López, S. (2010). Historia de vida de buenos profesores: experiencia e impacto en las aulas. Profesorado. Revista de Currículum y Formación de Profesorado, 14(3), 149164. Recuperado de https://www.redalyc. org/pdf/567/56715702011.pdf

Morán, P. (2004). La docencia como recreación y construcción del conocimiento Sentido pedagógico de la investigación en el aula. Perfiles educativos, 26(105-106), 41-72. Recuperado de http://www. scielo.org. $\mathrm{mx} / \mathrm{scielo}$. php? pid=S018526982004000100003\&script=sci_arttext

Pérez, L., y Pérez, Á. (2015). Los Estándares de Finlandia en las pruebas de selección inicial de profesorado en España. Revista Interuniversitaria de Formación del Profesorado, 29(2), 119141. Recuperado de https://www.redalyc. org/html/274/27443659009/

Tannenbaum, A. (1986). Giftedness: A psychosocial approach. In R. Sternberg y J. Davidson (Eds.), Conceptions of giftedness (pp. 21-252). New York: Cambridge University Press.

Tannenbaum, A. (1997). The Meaning and Making of Giftedness. En N. Colangelo y G. Davis (Eds.), Handbook of Gifted Education (pp. 27-42) Boston: Allyn and Bacon.

Urbina, J. (2015). Maestros de la pasión por aprender "cuchillas, pero chéveres". Revista Colombiana de Educación, (68), 91-111. Recuperado de https://www.redalyc.org/ pdf/4136/413638648005.pdf 